\title{
MAXIMAL IDEAL SPACES AND $A$-CONVEXITY
}

\author{
D. R. WILKEN ${ }^{1}$
}

1. Introduction. Let $X$ be a compact Hausdorff space. $C(X)$ denotes the Banach algebra of all complex valued continuous functions on $X$ with the supremum norm. A subalgebra $A$ contained in $C(X)$ is called a function algebra on $X$ if it satisfies the following conditions.

(i) $A$ separates points on $X$.

(ii) The constant functions are in $A$, i.e., 1 belongs to $A$.

(iii) $A$ is closed in $C(X)$.

For a function algebra $A$ the space of maximal ideals is denoted by $M_{A}$ and the Silov boundary by $S_{A}$. Both $S_{A}$ and $M_{A}$ carry natural compact Hausdorff topologies and $S_{A}$ and $X$ can be imbedded in $M_{A}$ so that, identifying $S_{A}$ and $X$ with their images in $M_{A}$, we have $S_{A} \subset X \subset M_{A}$. Moreover $A$, via restriction, is a function algebra on $S_{A}$ and, via the Gelfand representation, extends to a function algebra on $M_{A} . S_{A}$ and $M_{A}$ are respectively the "smallest" and "largest" compact Hausdorff spaces on which $A$ can be realized as a function algebra.

If $A$ and $B$ are function algebras on $M_{A}$ with $A$ a subalgebra of $B$, frequently it is of interest to know when the maximal ideal space of $B$ coincides with that of $A$. For example, this is the case when one wishes to apply the local maximum modulus principle to $B$. Thus, when considering a function algebra $B$ as above, it would be helpful to have criteria for determining the relation of $M_{B}$ to $M_{A}$. In this direction Stolzenberg [4] has shown that if the functions in $B$ agree locally with functions in $A$, then $M_{B}$ coincides with $M_{A}$. The main purpose of this note is to give a method of determining $M_{B}$ explicitly in terms of $M_{A}$ in many cases, and to present a direct way of constructing function algebras $B$ as above with larger maximal ideal spaces. Included is a counterexample to a question apparently raised by Hoffman and referred to by Glicksberg in [1].

2. $A$-convexity. For an arbitrary function algebra $A$ with maximal ideal space $M_{A}$ we use $A_{F}$ to denote the uniform closure on a closed set $F$ in $M_{A}$ of the restriction algebra of $A$ to $F$.

2.1. Definition. For a closed set $F$ in $M_{A}$, the $A$-convex hull of $F$ in $M_{A}$, denoted $M_{F}$, is

Received by the editors January 6, 1966.

1 This research was supported in part by NSF Grant GE-6587 and in part by a NSF Postdoctoral Fellowship. 


$$
M_{F}=\left\{x_{*} \in M_{A}:|g(x)| \leqq\|g\|_{F}, \text { for all } g \in A\right\}
$$

$\left(\|g\|_{F}=\sup _{F}|g|\right) . F$ is said to be $A$-convex if $M_{F}=F$.

Thus $M_{F}$ is the maximal ideal space of the function algebra $A_{F}$ (cf. [3]) and $F$ is $A$-convex if and only if $M_{A_{F}}=F$. For a finitely generated algebra $A$, the concept of $A$-convexity coincides with that of polynomial convexity in the space of $n$-complex variables.

2.2. Theorem. Let $A$ be a function algebra on $M_{A}$, let $f$ be in $C\left(M_{A}\right)$ and let $B$ be the function algebra generated by $A$ and $f$ on $M_{A}$. Let $R(f)$ denote the range of $f$ on $M_{A}$. For $z$ in $R(f)$, let $T_{z}=\left\{x \in M_{A}: f(x)=z\right\}$. Then either $T_{z}$ is $A$-convex for every $z$ in $R(f)$ or $M_{B}$ properly contains $M_{A}$. (Since $B$ is a function algebra on $M_{A}, M_{A}$ is imbedded in $M_{B}$. It is in terms of this imbedding that the final statement of the theorem is to be interpreted.)

Proof. Let $M_{z}$ denote the $A$-convex hull of $T_{z}$ in $M_{A}$. Let $K \subset M_{A} \times C$ be defined by $K=\left\{(x, f(x)): x \in M_{A}\right\}$. (C denotes the complex plane.) Then $K$ is an imbedding of $M_{A}$ in $M_{A} \times C$. Let

$$
H=\left\{(x, w) \in M_{A} \times C: x \in M_{w}\right\} .
$$

We claim that $H$ can be imbedded in $M_{B}$. Let $r$ denote the canonical retraction of $M_{B}$ on to $M_{A}$, i.e., $r: M_{B} \rightarrow M_{A}$ satisfies $x=r(y)$ if and only if $g(x)=g(y)$ for all $g$ in $A$. (Since $A \subset B$, the functions in $A$ extend continuously to functions on $M_{B}$.) Define a mapping $s: M_{B} \rightarrow M_{A}$ $\times C$ by $s(m)=(r(m), f(m))$. We show that $s$ is an imbedding of $M_{B}$ into $M_{A} \times \boldsymbol{C}$ such that $H \subset s\left(M_{B}\right)$.

(i) $s$ is one-to-one. If $s\left(m_{1}\right)=s\left(m_{2}\right)$, then $r\left(m_{1}\right)=r\left(m_{2}\right)$ so that $g\left(m_{1}\right)=g\left(r\left(m_{1}\right)\right)=g\left(r\left(m_{2}\right)\right)=g\left(m_{2}\right)$ for all $g$ in $A$. Also $f\left(m_{1}\right)=f\left(m_{2}\right)$. Hence $h\left(m_{1}\right)=h\left(m_{2}\right)$ for all $h$ in $B$ and $m_{1}=m_{2}$.

(ii) $H \subset s\left(M_{B}\right)$. For $(x, w) \in H$ we define a multiplicative functional $m(x, w)$ on $B$ as follows. Let $B^{\prime}$ be the dense subalgebra of $B$ of all polynomials in $f$ with coefficients from $A$. Define $m(x, w)$ on $B^{\prime}$ by

$$
m(x, w)\left(\sum a_{i} f^{i}\right)=\sum a_{i}(x) w^{i}, \quad a_{i} \in A .
$$

Then $m(x, w)$ is clearly a multiplicative linear functional on $B^{\prime}$. To extend it to $B$ it suffices to show $m(x, w)$ is bounded on $B^{\prime}$. But

$$
\left|m(x, w)\left(\sum a_{i} f^{i}\right)\right|=\left|\sum a_{i}(x) w^{i}\right| \leqq\left\|\sum a_{i} w^{i}\right\|_{T_{w}}=\left\|\sum a_{i} f^{i}\right\|_{T_{w}}
$$

since $\sum a_{i} w^{i} \in A$ and $(x, w) \in H$, i.e., $x \in M_{w}$. Thus $m(x, w)$ extends to a multiplicative functional on $B$. Also for $g$ in $A, m(x, w)(g)=g(x)$ implies $r(m(x, w))=x$; and $m(x, w)(f)=w$. Therefore $s(m(x, w))$ $=(x, w)$ and $H \subset s\left(M_{B}\right)$. 
(iii) $s\left(M_{A}\right)=K$. For $m \in M_{A} \subset M_{B}, r(m)=m$. Hence $s(m)=(m, f(m))$ $\in K$. If we now identify $M_{A}$ and $M_{B}$ with their imbeddings under $s$ in $M_{A} \times C$ we have $M_{A} \subset H \subset M_{B}$. To obtain the theorem we need now merely observe that if $T_{z}$ is not $A$-convex for some $z$, then $M_{z}$ properly contains $T_{z}$ so that $H$, and hence $M_{B}$, properly contains $M_{A}$.

Evidently the theorem gives a direct method of exhibiting function algebras $A$ and $B$ satisfying $A \subset B \subset C\left(M_{A}\right)$ and with $M_{B}$ properly larger than $M_{A}$. We can also show that in many cases the set $H$ described in the proof of the theorem actually fills out $M_{B}$ so that we have an explicit description of $M_{B}$ in terms of $M_{A}$. To this end we prove the following lemma.

2.3. Lemma. Let $A, f$, and $B$ be as in Theorem 2.2. If $f\left(M_{B}\right)$ properly contains $f\left(M_{A}\right)$, then each point $w$ in $f\left(M_{B}\right)-f\left(M_{A}\right)$ lies in a bounded component of $\boldsymbol{C}-f\left(M_{A}\right)$. Moreover $f\left(M_{B}\right)$ is the union of $f\left(M_{A}\right)$ with those bounded components of $\mathrm{C}-f\left(M_{A}\right)$ which meet $f\left(M_{B}\right)$.

(In other words $f\left(M_{A}\right)$ can only be enlarged to $f\left(M_{B}\right)$ by completely filling in some holes.)

Proof. Let $m \in M_{B}$ be such that $f(m)$ is not in $f\left(M_{A}\right)$. If $f(m)$ lies in the unbounded component of $C-f\left(M_{A}\right)$, then there is a polynomial $p$ in one complex variable such that $|p(f(m))|>\|p\|_{f\left(M_{\boldsymbol{A}}\right)}$. Since $g=p(f)$ is an element of $B$ we have $|g(m)|>\|g\|_{M_{A}}$. This contradicts the fact that $B$ as a function algebra on $M_{A}$ has its Silov boundary contained in $M_{A}$. To finish the lemma suppose $f(m)$ lies in some bounded component $U$ of $\boldsymbol{C}-f\left(M_{A}\right)$. If $U$ is not contained in $f\left(M_{B}\right)$ there is a point $w$ on the boundary of $f\left(M_{B}\right)$ and contained in $U-f\left(M_{A}\right)$. Let $w=f\left(m_{0}\right)$. Choose $a$ in $U-f\left(M_{B}\right)$ such that

$$
|a-w|<\inf _{m^{\prime} \in M_{A}}\left|w-f\left(m^{\prime}\right)\right| .
$$

Then $g(z)=1 /(z-a)$ is analytic on a neighborhood of $f\left(M_{B}\right)$ so that $g(f) \in B$. But $\left|g\left(f\left(m_{0}\right)\right)\right|>\|g(f)\|_{M_{A}}$, which, as above, is impossible.

2.4. Theorem. Let $H$ be as described in the proof of Theorem 2.2, i.e., $H=\left\{(x, w) \in M_{A} \times C: x \in M_{w}\right\}$. If $f\left(M_{A}\right)$ is a compact, nonseparating subset of the plane without interior, then $M_{B}=H$.

Proof. By Lemma 2.3, $f\left(M_{B}\right)=f\left(M_{A}\right)$. As in Theorem 2.2 consider the imbedding $s: M_{B} \rightarrow M_{A} \times C$ given by $s(m)=(r(m), f(m))$ where $r$ is the canonical retraction of $M_{B}$ onto $M_{A}$. Then

$$
s\left(M_{A}\right) \subset H \subset s\left(M_{B}\right) \subset M_{A} \times f\left(M_{A}\right)
$$

and we wish to show $H=s\left(M_{B}\right)$. If $s(m) \in s\left(M_{B}\right)-H$, from the definition of $H$, there exists a function $g$ in $A$ such that $1=|g(r(m))|$ 
$>\|g\|_{M_{f(m)}}$. Since $s(m) \notin s\left(M_{A}\right)$ we can choose a neighborhood $V$ of $f(m)$ in $C$ such that

$$
(t, \beta) \in s\left(M_{A}\right) \cap\left[M_{A} \times\left(V \cap f\left(M_{A}\right)\right] \Rightarrow|g(t)|<|g(r(m))| .\right.
$$

Also since $f\left(M_{A}\right)$ is a compact nonseparating subset of $\boldsymbol{C}$ without interior every continuous function on $f\left(M_{A}\right)$ can be uniformly approximated on $f\left(M_{A}\right)$ by polynomials. In particular if $h$ is a function which peaks at $f(m)$, i.e.,

$$
h(f(m))=1,|h(z)|<1 \text { for } z \in f\left(M_{A}\right), z=f(m),
$$

then $h$ can be approximated by polynomials. But $f\left(M_{A}\right)=f\left(M_{B}\right)$ implies $h(f)$ is in $B$. Choose an integer $N$ such that $\left\|h^{N}\right\|_{f\left(M_{A}\right)-V}<1 /\|g\|$. Let $h^{\prime}=h^{N}(f) g$. Then $h^{\prime} \in B,\left|h^{\prime}(m)\right|=1$ and $\left\|h^{\prime}\right\|_{M_{A}}<1$, a contradiction as in Lemma 2.3 .

2.5. Corollary. If $f$ is real-valued on $M_{A}$, then $M_{B}=H$.

Each of the above statements has an immediate extension to a more general situation in which a finite number of functions are adjoined to $A$. Then the statements of the results have to be formulated in terms of intersections of the level sets of the functions. As an indication we formulate Corollary 2.5 in the more general setting. Let $f_{1}, f_{2}, \cdots, f_{n} \in C\left(M_{A}\right)$, let

$$
\begin{aligned}
T\left(w_{1}, \cdots, w_{n}\right) & =\left\{x \in M_{A}: f_{i}(x)=w_{i}, i=1,2, \cdots, n\right\}, \\
M\left(w_{1}, \cdots, w_{n}\right) & =\text { the } A \text {-convex hull of } T\left(w_{1}, \cdots, w_{n}\right)
\end{aligned}
$$

and

$$
H=\left\{\left(x, w_{1}, \cdots, w_{n}\right) \in M_{A} \times C^{n}: x \in M\left(w_{1}, \cdots, w_{n}\right)\right\} .
$$

2.5'. Corollary. If $f_{1}, f_{2}, \cdots, f_{n}$ are real-valued on $M_{A}$, and $B$ is the function algebra generated by $A$ and $f_{1}, f_{2}, \cdot \cdot, f_{n}$ on $M_{A}$, then $M_{B}=H$.

Corollary 2.5 contains as a special case an observation made by Mergelyan [2] concerning the disc algebra. He observed that the set of maximal ideals of the algebra generated by the polynomials and a real function on the unit disc in the complex plane coincides with the disc just in the case that the level sets of the real function do not divide the plane.

3. Examples. The results above give an explicit way of relating the maximal ideal spaces of certain algebras and one naturally inquires whether, in the situations considered, it is possible to deduce 
that this relation always obtains. That is, in the terminology of $\$ 2$, is it always the case that $M_{B}=H$ ? In this section we describe some simple examples which show that Theorem 2.4 cannot be essentially strengthened. We also include the counterexample mentioned in the introduction.

3.1. Example. Let $D=\{z \in \mathrm{C}:|z| \leqq 1\}, \Gamma=\{z:|z|=1\}$ and let $A$ be the disc algebra consisting of the uniform limits of polynomials on $D$. Let $f \in C(D)$ be given by $f(z)=\exp (2 \pi i|z|)$, and let $B$ be the function algebra generated by $A$ and $f$ on $D$. That is, if $f_{0}$ is the identity function on $D, f_{0}(z)=z$, then $B$ is the algebra generated by $f$ and $f_{0}$. We have $A \subset B \subset C(D), M_{A}=D$, and $f\left(M_{A}\right)=\Gamma$ is a compact subset of $C$ without interior. For $w \in f\left(M_{A}\right), w=\exp (2 \pi i t)$,

$$
T_{w}=\{z \in D: f(z)=w\}=\{z:|z|=t\} .
$$

Hence $M_{w}=\{z \in D:|z| \leqq t\}$, the $A$-convex hull of $T_{w}$ and

$$
H=\left\{(z, w) \in D \times C: z \in M_{w}\right\}=\{(z, \exp (2 \pi i t)): 0 \leqq|z| \leqq t \leqq 1\} .
$$

Let $s: M_{A} \rightarrow C^{2}$ be defined by $s(z)=(z, \exp (2 \pi i|z|))$ and consider the following subsets of $D \times D C C \times C=C^{2}$ :

$$
\begin{aligned}
& X=s(D)=\{z, \exp (2 \pi i|z|)):|z| \leqq 1\}, \\
& Y=\text { the polynomially convex hull of } X \text { in } C^{2} .
\end{aligned}
$$

Then $Y$ coincides with the maximal ideal space of $B$ and $X \subset H \subset Y$. We assert that $Y$ is properly larger than $H$. To see this it suffices to show $(0,0) \in Y$. But if $p\left(w_{1}, w_{2}\right)$ is any polynomial on $\boldsymbol{C}^{2}$, then

$$
|p(0,0)| \leqq \sup _{\left|w_{2}\right|=1}\left|p\left(0, w_{2}\right)\right| \leqq \sup _{X}\left|p\left(w_{1}, w_{2}\right)\right| .
$$

It is not difficult to see that, in fact, $Y=H \cup E$ where $E=\left\{\left(0, w_{2}\right)\right.$ : $\left.\left|w_{2}\right| \leqq 1\right\}$ and that the rationally convex hull of $X$ in $C^{2}$ is $H$. Thus this simple example also exhibits the interesting phenomenon of a disc in $\boldsymbol{C}^{2}$ whose rationally convex hull lies properly between itself and its polynomially convex hull. Geometrically $H$ looks like a solid cone bent around so that its tip touches the center of its base.

To give an example such that $f\left(M_{A}\right)$ has interior (but does not separate the plane) in which $M_{B}$ differs from $H$, take $A$ as the polynomial algebra on two disjoint discs in the plane, and adjoin the function which is the identity on the first disc and maps the second disc onto the boundary of the first.

Our final example is a counterexample to the following question (see $[1])$ : 
3.2. Question. Let $A \subset B \subset C\left(M_{A}\right)$ where $A$ and $B$ are function algebras with the same Silov boundary. Is $M_{B}=M_{A}$ ?

We give a negative reply which is a modification of a special case of Corollary 2.5 according to the observation that for any function algebra $A$ on $M_{A}$ it is possible to construct a function algebra $A^{\prime}$ satisfying:

(i) $M_{A}{ }^{\prime}=S_{A}{ }^{\prime}$, i.e., the Silov boundary and the maximal ideal space of $A^{\prime}$ coincide.

(ii) There is an imbedding of $M_{A}$ in $M_{A}^{\prime}$ such that $A^{\prime} \mid M_{A}=A$. To obtain this let $I$ be the unit interval and consider $M_{A}$ as identified with the subset $M_{A} \times\{0\}$ of $M_{A} \times I$. Extend $A$ to $A^{\prime}$ on $M_{A} \times I$ by setting

$$
A^{\prime}=\left\{f \in C\left(M_{A} \times I\right): f \mid\left(M_{A} \times\{0\}\right) \in A\right\} .
$$

Direct verification shows $M_{A}{ }^{\prime}=M_{A} \times I=S_{A^{\prime}}$.

3.3. Example. Let $A$ and $D$ be as in Example 3.1. Let $f(z)=|z|$ and let $B$ be the function algebra generated by $A$ and $f$ on $D$. Then by Corollary $2.5, M_{B}$ is the cone

$$
M_{B}=\{(z, t): 0 \leqq|z| \leqq t \leqq 1\} \subset C^{2},
$$

so that $M_{B}$ properly contains $M_{A}$. Let $A^{\prime}$ be constructed from $A$ as above, i.e.,

$$
A^{\prime}=\{f \in C(D \times I): f \mid(D \times\{0\}) \in A\}
$$

and let

$$
B^{\prime}=\{f \in C(D \times I): f \mid(D \times\{0\}) \in B\} .
$$

Then $M_{A^{\prime}}=M_{A} \times I=S_{A^{\prime}}=S_{B}{ }^{\prime}, A^{\prime} \subset B^{\prime} \subset C\left(M_{A^{\prime}}\right)$, but $M_{B}{ }^{\prime}$ is the cylinder with the bottom slice replaced by the cone which we have identified with $M_{B}$. Thus $M_{B}{ }^{\prime}$ properly contains $M_{A}{ }^{\prime}$.

\section{REFERENCES}

1. I. Glicksberg, Maximal algebras and a theorem of Rado, Pacific J. Math. 14 (1964), 919-941.

2. S. N. Mergelyan, Uniform approximations to functions of a complex variable, Uspehi Mat. Nauk 7 (1952), no. 2 (48), 31-122; English transl., Amer. Math. Soc. Transl. 101 (1954), 19.

3. H. Rossi, The local maximum modulus principle, Ann. of Math. 72 (1960), 1-11.

4. G. Stolzenberg, The maximal ideal space of the functions locally in a function algebra, Proc. Amer. Math. Soc. 14 (1963), 342-345.

Tulane UNIVERsity AND

BRANDEIS UNIVERSITY 